\title{
Calculation of ternary interdiffusion coefficients using a single diffusion couple
}

\author{
J. Cermak*, V. Rothova \\ Institute of Physics of Materials, v.v.i., AS CR, CEITEC IPM, Zizkova 22, CZ-61662 Brno, Czech Republic
}

Received 20 May 2015, received in revised form 3 December 2015, accepted 14 January 2016

\begin{abstract}
In this paper, the simple numerical algorithm is proposed that enables to estimate ternary interdiffusion coefficients $D$ in systems with not too strongly interacting components. $D$ 's are obtained from measurement with a single model diffusion couple. They can be used to predict concentration curves $C_{i}(x)(i=1,2,3)$ in arbitrary time with numerical errors that are comparable with experimental errors of measurement of $c_{i}$ itself and comply with theoretical constraints following from the linear irreversible thermodynamics. The algorithm does not need any external thermodynamic data and external measurements (e.g., it is not necessary to measure the shift of inert markers in the interface). It can be applied using commonly accessible SW, and it is not time-consuming. Values of $D$ are close to those obtained with the help of much more laborious experimental - also approximate - methods.
\end{abstract}

K e y w o r d s: diffusion, interdiffusion, ternary alloys, transport properties, ternary diffusion coefficients

\section{Introduction}

There are many processes in technical practice that are controlled by chemical diffusion. To describe the kinetics of these phenomena, which enables, e.g., estimation of optimum production of construction components or prediction of their behavior during the operation, it is necessary to know concentration and temperature dependent interdiffusion coefficients $D$. Since there is no general theory of $D$ 's, their values must be obtained by extremely laborious experiments with many diffusion couples.

Mutual diffusion in an $n$-component system can be described by the formalism of linear irreversible thermodynamics (LIT). The central problem with the general approach, applied to this case, is how to find diffusion coefficients $\tilde{D}_{i j}^{(3)}(i, j=1,2$ are two independent components, the third one - the upper index - is dependent) that relate diffusion fluxes of chemical components to concentration gradients. These interdiffusion coefficients depend on temperature and on the local composition $c_{i}$. It is not a simple task - even with constant diffusion coefficients - to find a complete set of $\bar{D}_{i j}^{(3)}$ 's, because the number of
$\bar{D}_{i j}^{(3)}$ 's exceeds the number of equations that offers LIT.

Astonishingly, evaluation of $\bar{D}_{i j}^{(3)}$ 's is still an open problem, therefore, considerable effort has been devoted to the elaboration of approximate techniques of calculation and/or estimation of interdiffusion coefficients [1-13]. In the simplest cases, the modified Boltzmann-Matano (BM) [1, 2] and Hall's (HM) [3] analyses were applied. In all other instances, special evaluation procedures [5] or further independent equations from outside of LIT (e.g., observation of the motion of inert markers [6]) are needed. Transfer-matrix method developed for analysis of multicomponent diffusion [7] is based on a partition of diffusion zone into segments, where the diffusion coefficients are constant. Completely different is pragmatic method recently developed by Chinese group [8-10]. Method is based on the phenomenological theory of diffusion that uses external thermodynamic data.

A rigorous solution of the problem in ternary systems $(n=3)$, completely within the frame of LIT, is the Matano-Kirkaldy's method (MK) [5] utilizing two diffusion couples with intersecting diffusion paths. This is, however, a very troublesome procedure, since

\footnotetext{
*Corresponding author: tel.: 00420 532290422; fax: 00420 541218657; e-mail address: cermak@ipm.cz
} 
at least $m+1$ diffusion couples must be analyzed to obtain $m$ sets of diffusion coefficients at $m$ different compositions. Moreover, there are some theoretical and experimental suggestions that such coefficients may depend on the choice of intersecting diffusion paths $[11,12]$.

The idea used in the present paper was inspired by works by Dayananda's group (see, e.g., [13]) that made an accessible evaluation of interdiffusion coefficients using a single diffusion couple. In the present paper, revised and extended method [14] of estimation of $\tilde{D}_{i j}^{(3)}$ from a single diffusion couple is proposed. The detailed evaluation procedure is illustrated using our previous experimental data for the quasi-binary diffusion couple $\mathrm{Ni}_{3} \mathrm{Al}-\mathrm{Cr} / \mathrm{Ni}_{3} \mathrm{Al}$. Our results are also compared with BM, HM and with data calculated from other - more complicated - numeric algorithm MultiDiFlux [13, 15-17].

\section{Theory}

\subsection{Principal idea}

Dayananda and Sohn [18] published equation that relates the diffusion flux $J_{i}$ to concentration curves $C_{i}(x)$ via bypassing the Fick's law, and consequently standing outside of LIT. The fundamental idea of the authors was the introduction of forms

$$
I_{i}^{p}=\int_{x_{\mathrm{L}}}^{x_{\mathrm{R}}} J_{i}\left(x-x_{\mathrm{m}}\right)^{p} \mathrm{~d} x, p=0,1, \ldots
$$

that produced other independent equations suitable for calculation of an average value of independent interdiffusion coefficients $\bar{D}_{i j}^{(3)}$. Within a selected interval in the segment of the diffusion zone limited by co-ordinates $x_{\mathrm{L}}$ and $x_{\mathrm{R}}$, the $\bar{D}_{i j}^{(3)}$ 's were considered constants.

In Eq. (1), the index $i$ enumerates components, the exponent $p=0,1, \ldots$ is an integer and $x_{m}$ refer to the position of the Matano plane calculated from equation

$$
\int_{-\infty}^{+\infty}\left(x-x_{m}\right) \mathrm{d} C_{i}=0
$$

for each component $i$. Values of $I_{i}^{p}$ in Eq. (1) can be obtained numerically using known diffusion fluxes $J_{i}$ calculated from relation [19]:

$$
\begin{aligned}
& J_{i}(x)=\frac{C_{i}^{-}-C_{i}^{+}}{2 t}\left\{Y_{i}(x) \int_{-\infty}^{x}\left[1-Y_{i}(x)\right] \mathrm{d} x+\right. \\
& \left.+\left[1-Y_{i}(x)\right] \int_{x}^{\infty} Y_{i}(x) \mathrm{d} x\right\}
\end{aligned}
$$

where the concentrations $C_{i}$ were transformed as follows [20]:

$$
Y_{i}(x)=\frac{C_{i}(x)-C_{i}^{+}}{C_{i}^{-}-C_{i}^{+}} .
$$

$C_{i}^{-}$and $C_{i}^{+}$are the terminal concentrations of the $i$-th component in $x=-\infty$ and $x=+\infty$, respectively, and $t$ is the diffusion time. The molar volume in Eq. (3) is assumed to be constant.

For the case of ternary interdiffusion $(n=3)$, there are four independent diffusion coefficients $\tilde{D}_{i j}^{(3)}$. Authors of paper [18] show that using $p=0$ and 1 , four independent equations can be obtained and solved yielding four average coefficients $\bar{D}_{i j}^{(3)}$. Within the interval $\Delta x=\left\langle x_{\mathrm{L}}, x_{\mathrm{R}}>\right.$, the values of $\bar{D}_{i j}^{(3)}$ are constant. Dayananda and Sohn [18] proposed that the procedure applies to relatively wide intervals $\Delta x$ only (hundreds of $\mu \mathrm{m})$.

\subsection{Our extension}

We have shown [14] that the original method introduced by Dayananda and Sohn [18] can be extended to the case where the diffusion zone is subdivided into very narrow intervals $\Delta x=x_{q+1}-x_{q}$ ( $\Delta x$ may decrease down to about $1 \mu \mathrm{m})$, it is possible to obtain a series of $\bar{D}_{i j}^{(3)}$ 's (one average $\bar{D}_{i j}^{(3)}$ value for each interval $\Delta x$ ) that approach the true interdiffusion coefficients $\tilde{D}_{i j}^{(3)}\left(C_{i}\right)$ within individual intervals.

The system of four independent equations can be formally obtained if both sides of the two equations for diffusion flux (LIT)

$$
J_{i}=-\sum_{j=1,2} \tilde{D}_{i j}^{(3)} \frac{\partial C_{j}}{\partial x}, i=1,2
$$

are multiplied by $\left(x-x_{m}\right)^{p}$ and then integrated over $\Delta x$. After rearrangement, the four equations read

$$
I_{i}^{0}=-\bar{D}_{i 1}^{(3)} \Delta C_{1}-\bar{D}_{i 2}^{(3)} \Delta C_{2}, i=1,2, p=0,
$$

and

$$
I_{i}^{1}=-\bar{D}_{i 1}^{(3)} \Delta J_{1}-\bar{D}_{i 2}^{(3)} \Delta J_{2}, i=1,2, p=1,
$$

if the following substitutions are used

$$
\begin{gathered}
\Delta C_{i}=C_{i}\left(x_{q+1}\right)-C_{i}\left(x_{q}\right), \\
\Delta J_{i}=J_{i}\left(x_{q+1}\right)-J_{i}\left(x_{q}\right) .
\end{gathered}
$$

Final relations for $D$ 's follow directly from Eqs. (6) and (7):

$$
\bar{D}_{11}^{(3)}=\frac{\Delta J_{2} I_{1}^{0}-\frac{\Delta C_{2}}{2 t} I_{1}^{1}}{M},
$$




$$
\begin{gathered}
\bar{D}_{12}^{(3)}=-\frac{\Delta J_{1} I_{1}^{0}-\frac{\Delta C_{1}}{2 t} I_{1}^{1}}{M}, \\
\bar{D}_{21}^{(3)}=\frac{\Delta J_{2} I_{2}^{0}-\frac{\Delta C_{2}}{2 t} I_{2}^{1}}{M},
\end{gathered}
$$

and

$$
\bar{D}_{22}^{(3)}=-\frac{\Delta J_{1} I_{2}^{0}-\frac{\Delta C_{1}}{2 t} I_{2}^{1}}{M},
$$

with the denominator

$$
M=\Delta C_{1} \Delta J_{2}-\Delta C_{2} \Delta J_{1}
$$

\section{Description of the numerical treatment}

\subsection{Raw data processing}

Smoothing the concentration profiles that removes the scatter of measured $C_{i}$ 's is the first step. In this work, trial functions from the TableCurve $2 D$ -Automated curve fitting and equation discovery [21] were used. This commercial SW package tests the fit quality of thousands of internal equation types and/or user-defined functions to measured data. As a fact of experience, the best candidate functions of $C_{i}(x)$ found by the SW must be carefully checked with respect to their physical meaning.

\subsection{Numerical calculation of $\bar{D}_{i j}^{(3)}$}

From the smoothed concentration profiles $C_{i}(x)$, the dimensionless concentrations $Y_{i}(x)$ can be calculated using Eq. (4) and diffusion flux $J_{i}$ and integrals $I_{i}^{p}$ from Eqs. (3) and (1), respectively. Of course, known analytic form of $C_{i}(x)$ would allow one to get $J$ 's and $I$ 's analytically, but for most practical purposes, numerical quadrature within each $\Delta x$ is sufficient. It can be easily found that ten integration nodes within each $\Delta x$ lead to reasonably correct results. Coefficients $\bar{D}_{i j}^{(3)}$ can be easily calculated from Eqs. (10)(13).

\subsection{Limit $\Delta x \rightarrow 0$}

It is obvious from Eq. (14) that coefficients $\bar{D}_{i j}^{(3)}$ show singular behavior if $M=0$, i.e., if

$$
\Delta C_{1} \Delta J_{2}=\Delta C_{2} \Delta J_{1}
$$

However, this can be avoided by a change of nodes $x_{\mathrm{q}}$.

Numerical instability may also occur if the width of interval $\Delta x$ decreases. It follows that differences $\Delta J_{i}$,
$\Delta C_{i}$ and integrals $I_{i}^{p}$ decrease as well and Eqs. (10)(14) approach the indeterminate expressions. In such a case, coefficients $\bar{D}_{i j}^{(3)}$ still can be evaluated, since fitting curves $C_{i}(x)$ are known in an analytic form and $D$ 's can be calculated using the known L'Hôpital's rule. Anyway, the numerical stability of $D$ 's can be improved by running the mathematical operations with sufficiently high numerical accuracy, which is not a compelling problem. It is worth noting at this place that the high numerical accuracy is needed for the mathematic operations with fitting functions $C_{i}(x)$. Of course, the attainable accuracy of primary data $C_{i}$ measured by electron probe microanalysis is much lower. The scatter of $C_{i}$ has to be suppressed by careful smoothing.

\section{Results}

\subsection{Evaluation of diffusion coefficients}

Application of the proposed method is illustrated on data from mutual diffusion in single-phase couple $\mathrm{Ni}_{3} \mathrm{Al}-\mathrm{Cr} / \mathrm{Ni}_{3} \mathrm{Al}$ annealed at $1473 \mathrm{~K} / 164 \mathrm{ks}$ [14]. The Ni-Al-based intermetallics (modified, e.g., by Ta, Mo [22], by $\mathrm{Ti}, \mathrm{Fe}[23]$, by Mo, Cr, V [24], etc.) are important for technical applications and hence, it is advisable to know the interdiffusion coefficients. Measured $C_{\mathrm{Cr}}$ and $C_{\mathrm{Al}}$, taken as independent concentrations, are plotted in Fig. 1 (points) together with fitted curves $C_{i}(x)$ (lines) and $99.99 \%$ confidence intervals (shaded area). The Al concentration profile was fitted by Gauss cumulative function [21] (solid line in Fig. 1a). To demonstrate a sensitivity of $\bar{D}_{i j}^{(3)}\left(C_{i}\right)$ to the choice of fitting function, the profile $C_{\mathrm{Cr}}(x)$ was fitted by an asymmetric sigmoid function [21] (fit 1, solid line in Fig. 1b) and by our user-defined function

$$
\begin{aligned}
& C_{\mathrm{Cr}}(x)=a_{1}\left\{\frac{1}{2}\left[1+\operatorname{erf}\left(\frac{x+a_{2}}{a_{3}}\right)\right] \operatorname{erfc}\left(\frac{x+a_{2}}{2 \sqrt{a_{4} t}}\right)+\right. \\
& \left.+\frac{1}{2}\left[1-\operatorname{erf}\left(\frac{x+a_{2}}{a_{3}}\right)\right] \operatorname{erfc}\left(\frac{x+a_{2}}{2 \sqrt{a_{5} t}}\right)\right\}
\end{aligned}
$$

where $a_{1}=1 / 2\left(C_{i}^{-}-C_{i}^{+}\right)$and $a_{2}-a_{5}$ are the fitting parameters (fit 2, dashed line in Fig. 1b). Both fitting functions coincide well one with another, except for the region of very small $C_{\mathrm{Cr}}$, as can be seen in the inset. Even in this region, the both functions lie within the confidence interval. Calculation of $\bar{D}_{i j}^{(3)}\left(C_{i}\right)$ from Eqs. (10)-(13) was a matter of elementary arithmetic.

Since the diffusion path $C_{i}=f\left(C_{j}\right)$ for the given diffusion couple is invariant during the diffusion time $t$, the chemical composition along the coordinate $x$ is completely and unambiguously described by one of the two independent concentrations $C_{i}$ only. Therefore, the final interdiffusion coefficients $\bar{D}_{i j}^{(3)}\left(C_{i}\right)$ for 

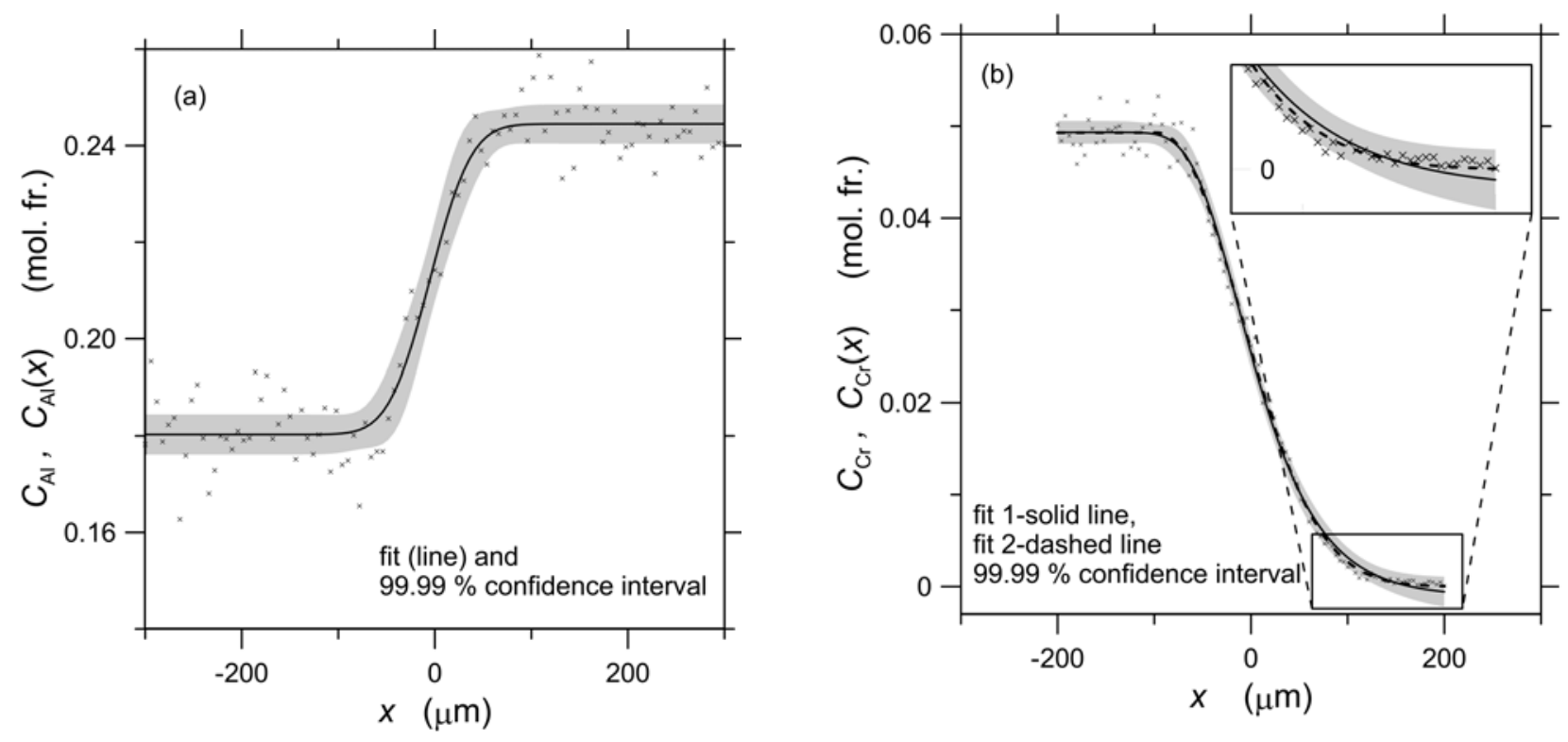

Fig. 1. Concentration profiles $C_{i}$ and fitting functions $C_{i}(x)$ measured in diffusion couple $\mathrm{Ni}_{3} \mathrm{Al}-\mathrm{Cr} / \mathrm{Ni}_{3} \mathrm{Al}$ after annealing at $1473 \mathrm{~K}$ for $164 \mathrm{ks}$ [14]: (a) - Al, (b) - Cr.

the given diffusion couple are presented in Figs. 2 and 3 in dependence on $C_{\mathrm{Cr}}$ only (solid lines). All the calculations were done easily with a numerical accuracy of 15 digits using Microsoft Excel.

A comparison of Fig. 2 for asymmetric sigmoid function (solid line) and Fig. 3 for user-defined function (solid line) in Eq. (16) shows that even a slight variation in $C_{i}(x)$ leads to a considerable change in resulting $D^{\prime}$ s.

\subsection{Inherent consistency}

Since the diagonal elements of diffusion matrix (with $i=j$ ) should be invariant against the choice of the third, dependent component, the calculation of ternary interdiffusion coefficients was done using $C_{\mathrm{Cr}}, C_{\mathrm{Al}}$ and measured concentration $C_{\mathrm{Ni}}$ to evaluate $\bar{D}_{\mathrm{CrCr}}^{(\mathrm{Al})}\left(C_{\mathrm{Cr}}\right)$ and $\bar{D}_{\mathrm{AlAl}}^{(\mathrm{Cr})}\left(C_{\mathrm{Cr}}\right)$. As it is shown in Figs. 2a,d, and Figs. 3a,d, these values (dash-and-dot lines) are reasonably close to $\bar{D}_{\mathrm{CrCr}}^{(\mathrm{Ni})}\left(C_{\mathrm{Cr}}\right)$ and $\bar{D}_{\mathrm{AlAl}}^{(\mathrm{Ni})}\left(C_{\mathrm{Cr}}\right)$ (solid lines), respectively, which complies with the needs of inherent consistency of the proposed method.

\subsection{Comparison with BM and $H M$}

The diffusion system $\mathrm{Ni}_{3} \mathrm{Al}-\mathrm{Cr} / \mathrm{Ni}_{3} \mathrm{Al}$ behaves almost as being quasi-binary. Therefore, we have made an attempt to evaluate coefficients of mutual diffusion $\tilde{D}_{\mathrm{Cr}}$ and $\tilde{D}_{\mathrm{Al}}$ by BM. One can see that the resulting $\tilde{D}_{i}$ 's , plotted in Figs. $2 \mathrm{a}, \mathrm{d}$, and $3 \mathrm{a}$, d in dependence on $C_{\mathrm{Cr}}$ (dashed lines), agree quite well with values $\bar{D}_{\mathrm{CrCr}}^{(k)}\left(C_{\mathrm{Cr}}\right)(k=\mathrm{Ni}, \mathrm{Al})$ and $\bar{D}_{\mathrm{AlAl}}^{(k)}\left(C_{\mathrm{Cr}}\right)(k=$
$\mathrm{Ni}, \mathrm{Cr}$ ), respectively. This can be considered as further evidence that values of $\bar{D}_{i j}^{(3)}\left(C_{i}\right)$ calculated by the present method lie close to those that could be expected. This conclusion is also supported by HM in terminal parts of the diffusion profile (see big full points in Figs. 2a,d, and Figs. 3a,d), where the BM is numerically unstable.

\subsection{Comparison with Multi DiFlux}

To compare the strength of our method, we also made calculation with the help of program MultiDiFlux [15, 16]. These values obtained with the same width of interval $\Delta x=2 \mu \mathrm{m}$ are shown in Figs. 2, 3 (crosses). It is obvious that our results are somewhat higher compared to results calculated with MultiDiFlux. However, values of $\bar{D}_{\mathrm{CrCr}}^{(\mathrm{Ni})}$ $\bar{D}_{\mathrm{CrAl}}^{(\mathrm{Ni})}$ and $\bar{D}_{\mathrm{AlCr}}^{(\mathrm{Ni})}$ calculated by our method in terminal parts of diffusion zone agree well with the respective values obtained by MultiDiFlux and agreement of values of $\bar{D}_{\mathrm{AlAl}}^{(\mathrm{Ni})}$ calculated by both methods can be seen in the middle of the diffusion zone.

\subsection{Dependence of D's on the interval width $\Delta x$}

It was shown previously [25] that values of $\bar{D}_{i j}^{(3)}\left(C_{i}\right)$ calculated with MultiDiFlux strongly depended on $\Delta x$. Hence, it suggests itself to test the stability of our method when the $\Delta x$ varies and to compare its behavior with that of MultiDiFlux in this aspect. 

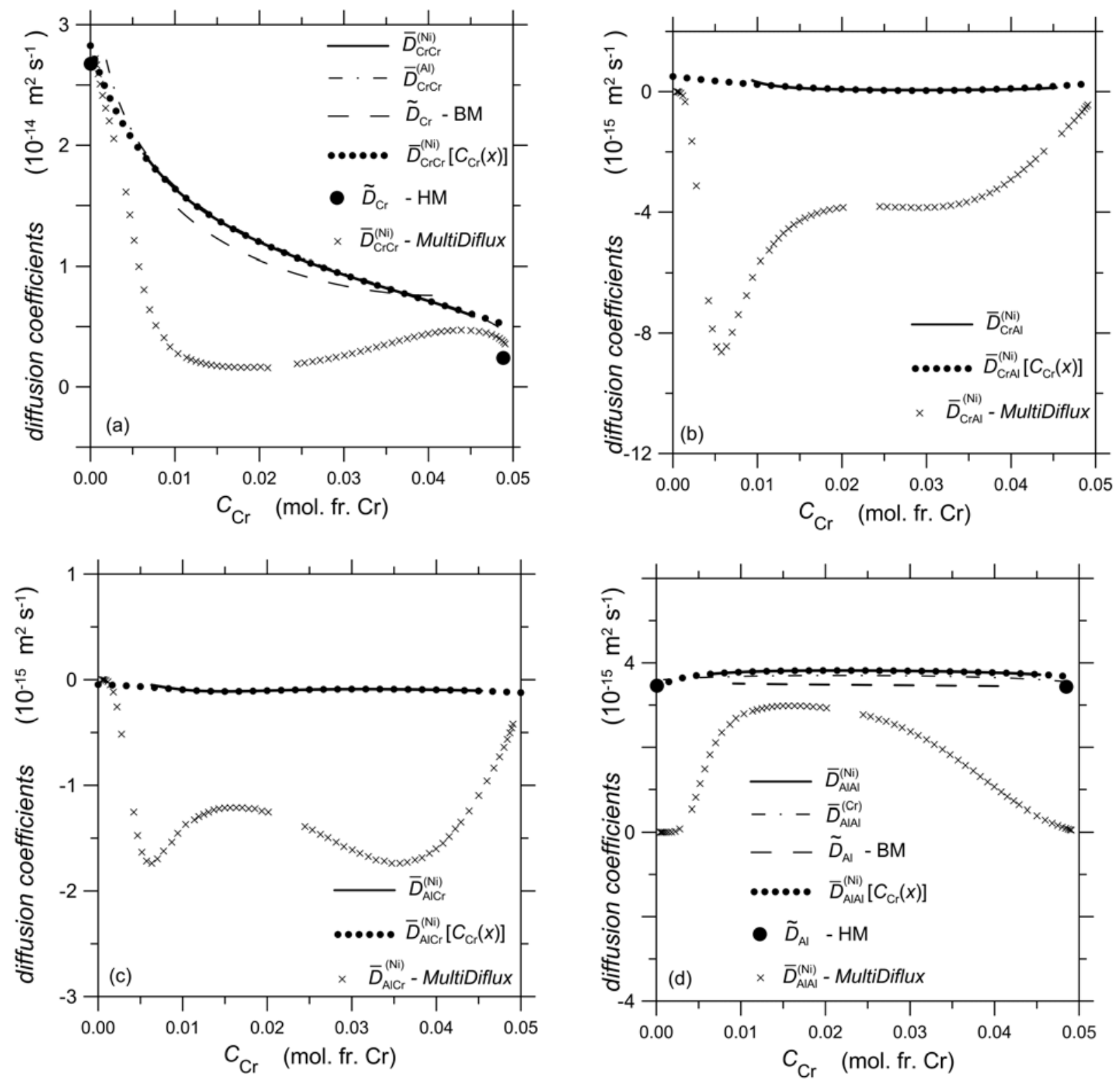

Fig. 2. Calculated ternary interdiffusion coefficients $\bar{D}_{i j}^{(k)}\left(C_{\mathrm{Cr}}\right)$ and interdiffusion coefficients in quasi-binary approximation, $\tilde{D}_{i}\left(C_{\mathrm{Cr}}\right)$ calculated by BM and HM; fit 1.

We varied $\Delta x$ from 2 to $32 \mu \mathrm{m}$ and calculated $\bar{D}_{i j}^{(\mathrm{Ni})}\left(C_{\mathrm{Cr}}\right)$ by the both methods. Results in Figs. 4 and 5 show that the sensitivity of our method to variation in $\Delta x$ is much weaker compared to the sensitivity of MultiDiFlux. Further, for greater $\Delta x$, the results from MultiDiFlux approach results from our method, especially in terminal parts of diffusion profile - see Fig. 5. The numerical instability of results from MultiDiFlux (see singularities in concentration dependence) and relatively high numerical sensitivity to $\Delta x$ may be the origin of differences between $D$ 's calculated by both methods for small $\Delta x$ (see Figs. 2, 3).

\subsection{Conditions of stability}

All values of $\bar{D}_{i j}^{(3)}\left(C_{i}\right)$ calculated by the present method satisfy stability constraints [26]:

$$
\begin{gathered}
\bar{D}_{i i}^{(3)}+\bar{D}_{j j}^{(3)}>0, \\
\bar{D}_{i i}^{(3)} \bar{D}_{j j}^{(3)}-\bar{D}_{i j}^{(3)} \bar{D}_{j i}^{(3)} \geq 0,
\end{gathered}
$$

and

$$
\left(D_{i i}^{(3)}-D_{j j}^{(3)}\right)^{2}-4 D_{i j}^{(3)} D_{j i}^{(3)} \geq 0
$$

within the diffusion zone. 

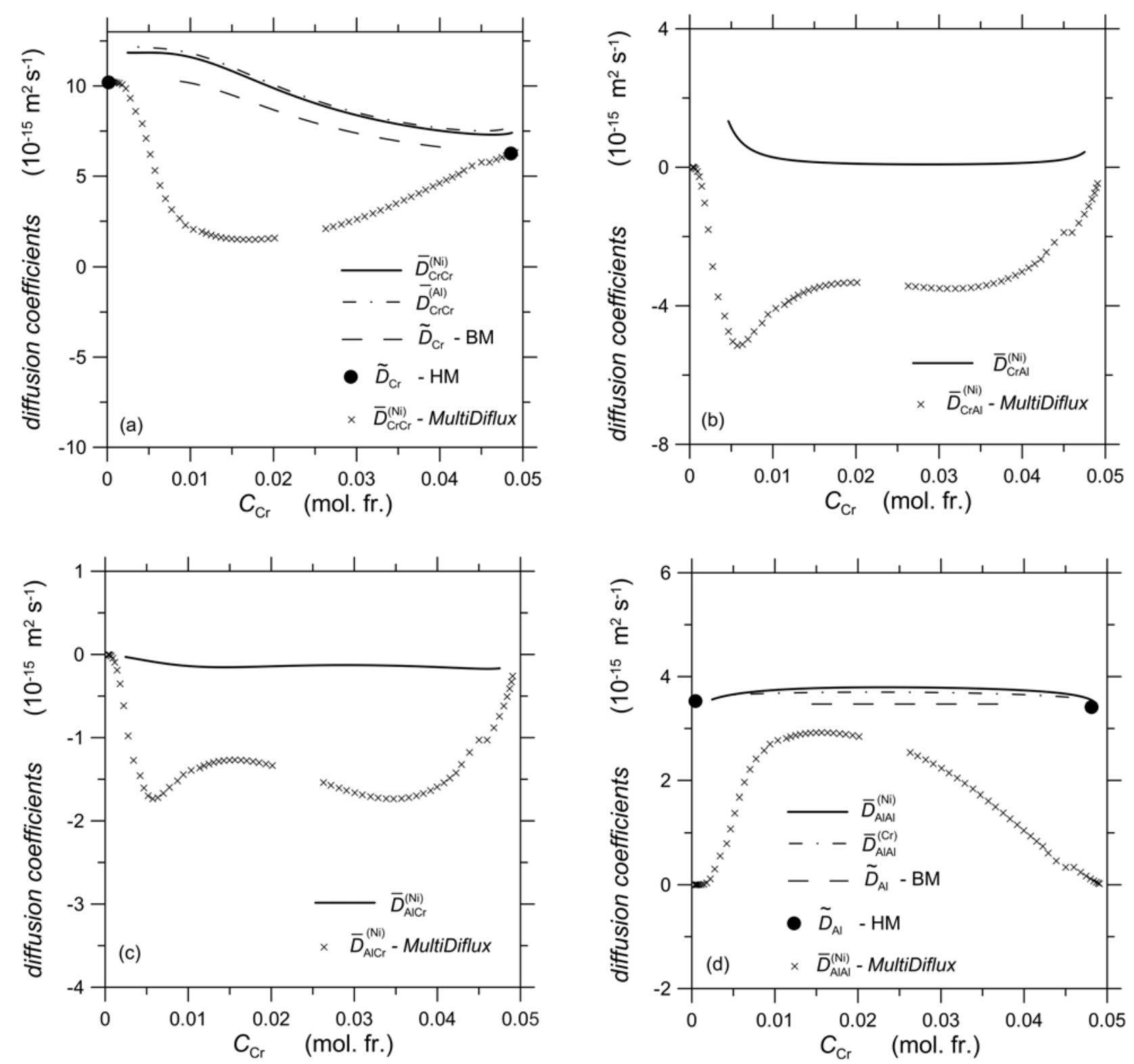

Fig. 3. Calculated ternary interdiffusion coefficients $\bar{D}_{i j}^{(k)}\left(C_{\mathrm{Cr}}\right)$ and interdiffusion coefficients in quasi-binary approximation, $\tilde{D}_{i}\left(C_{\mathrm{Cr}}\right)$ calculated by BM and HM; fit 2 .

\section{Reverse reconstructions of concentration profiles}

We compared concentration distribution $C_{i}(x, t)$, simulated using calculated values of $\bar{D}_{i j}^{(3)}\left(C_{i}\right)$, with measured concentrations $C_{i}$. It is easy to obtain concentrations $C_{i}(x, \tau)$ in successive iteration for diffusion times $\tau$ from 0 to $t$ : Starting from an initial distribution $C_{i}(x, 0)$ (e.g., very steep distribution of Gaussian type identical for both $i$ and $j$ ), concentration profiles for the next time period $\tau+\Delta \tau$ were calculated from recursive formula

$$
\begin{aligned}
& C_{i}(x, \tau+\Delta \tau)=C_{i}(x, \tau)+ \\
& +\frac{\Delta\left[\bar{D}_{i 1}^{(3)} \frac{\Delta C_{1}(x, \tau)}{\Delta x}+\bar{D}_{i 2}^{(3)} \frac{\Delta C_{2}(x, \tau)}{\Delta x}\right]}{\Delta x} \Delta \tau .
\end{aligned}
$$

For the iteration process, $D$ 's must be known for all $x$ in the diffusion zone. In terminal parts of diffusion couple, however, the calculated diffusion coefficients $\bar{D}_{i j}^{(3)}\left(C_{\mathrm{Cr}}\right)$ are not too reliable (solid lines are not plotted up to unaffected ends of diffusion couple - see 

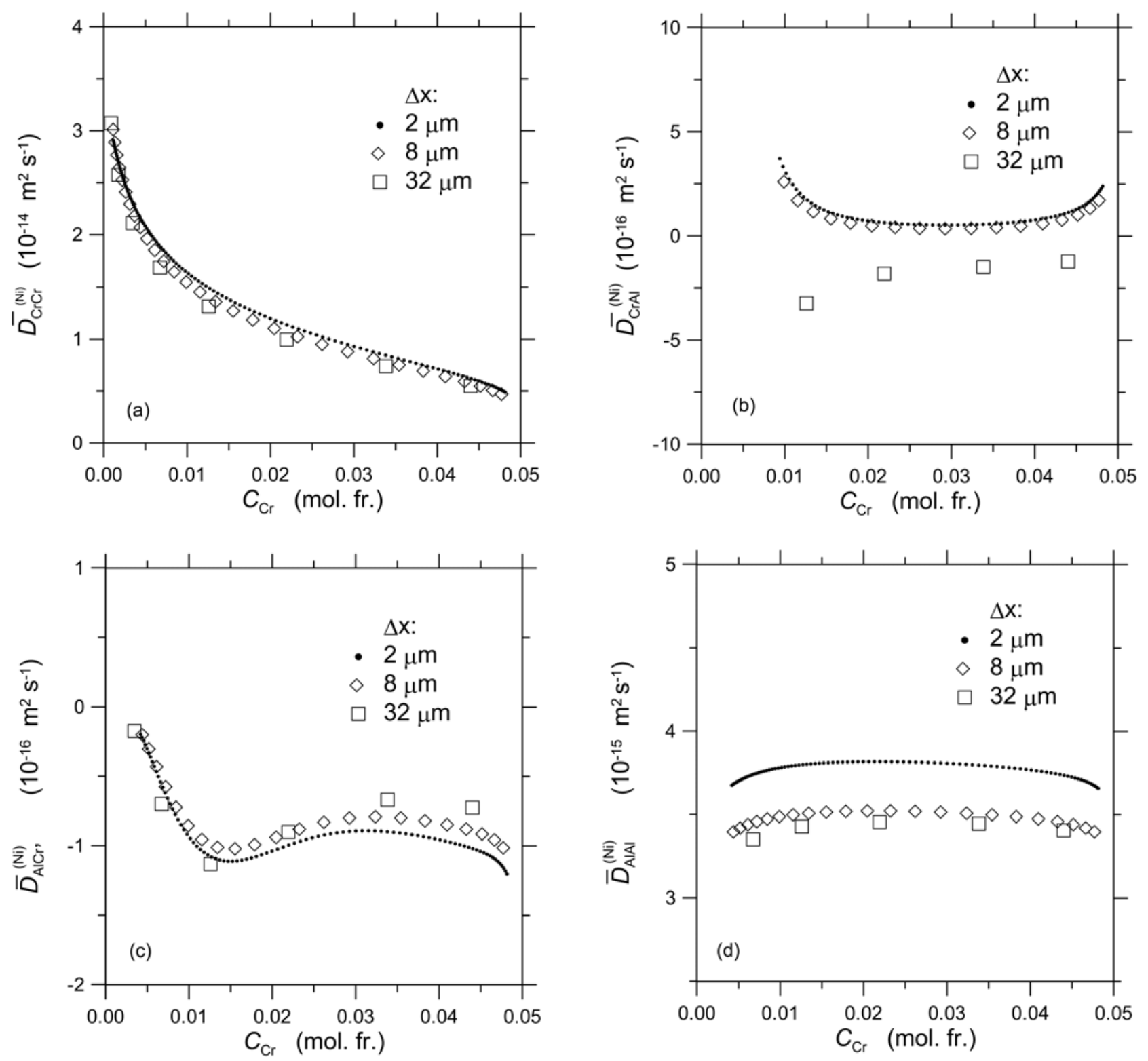

Fig. 4. Influence of $\Delta x$ on values of $\bar{D}_{i j}^{(k)}\left(C_{\mathrm{Cr}}\right)$ calculated by the proposed method; fit 1 .

Figs. 2-5). Therefore, we used - for the purpose of iterations - values $\bar{D}_{i j}^{(3)}\left[C_{\mathrm{Cr}}(x)\right]$ obtained by fitting the values of $\bar{D}_{i j}^{(3)}\left(C_{\mathrm{Cr}}\right)$ calculated within the central part of diffusion zone by high-precision rational function [21]

$$
\begin{aligned}
& \bar{D}_{i j}^{(3)}\left[C_{\mathrm{Cr}}(x)\right]= \\
& =\frac{a+c C_{\mathrm{Cr}}(x)+e C_{\mathrm{Cr}}^{2}(x)+g C_{\mathrm{Cr}}^{3}(x)+\cdots}{1+b C_{\mathrm{Cr}}+d C_{\mathrm{Cr}}^{2}(x)+\cdots} .
\end{aligned}
$$

Usually, using a few first terms is sufficient to fit well the calculated $D$ 's and also define reasonable values in terminal parts of diffusion couple. See examples in Fig. 2 - dotted lines. It should be emphasized that the knowledge of $\bar{D}_{i j}^{(3)}\left[C_{\mathrm{Cr}}(x)\right]$ values is necessary for iterations according to Eq. (20). Their accuracy in terminal parts of the couple is, however, not too important, since they stand in a product with small values of derivatives of $C_{i}$ (see the second term in Eq. (20)). Therefore, they do not contribute substantially to $C_{i}(x, \tau+\Delta \tau)$.

Results of the simulation are shown in Fig. 6. It is obvious that all simulated concentration profiles calculated with obtained $D$ 's agree very well with original data and moreover, they lie within the $99.99 \%$ confidence limits indicated by gray areas.

It is clearly seen that there is no significant difference between concentration profiles simulated with considerably different diffusion coefficients, as is 

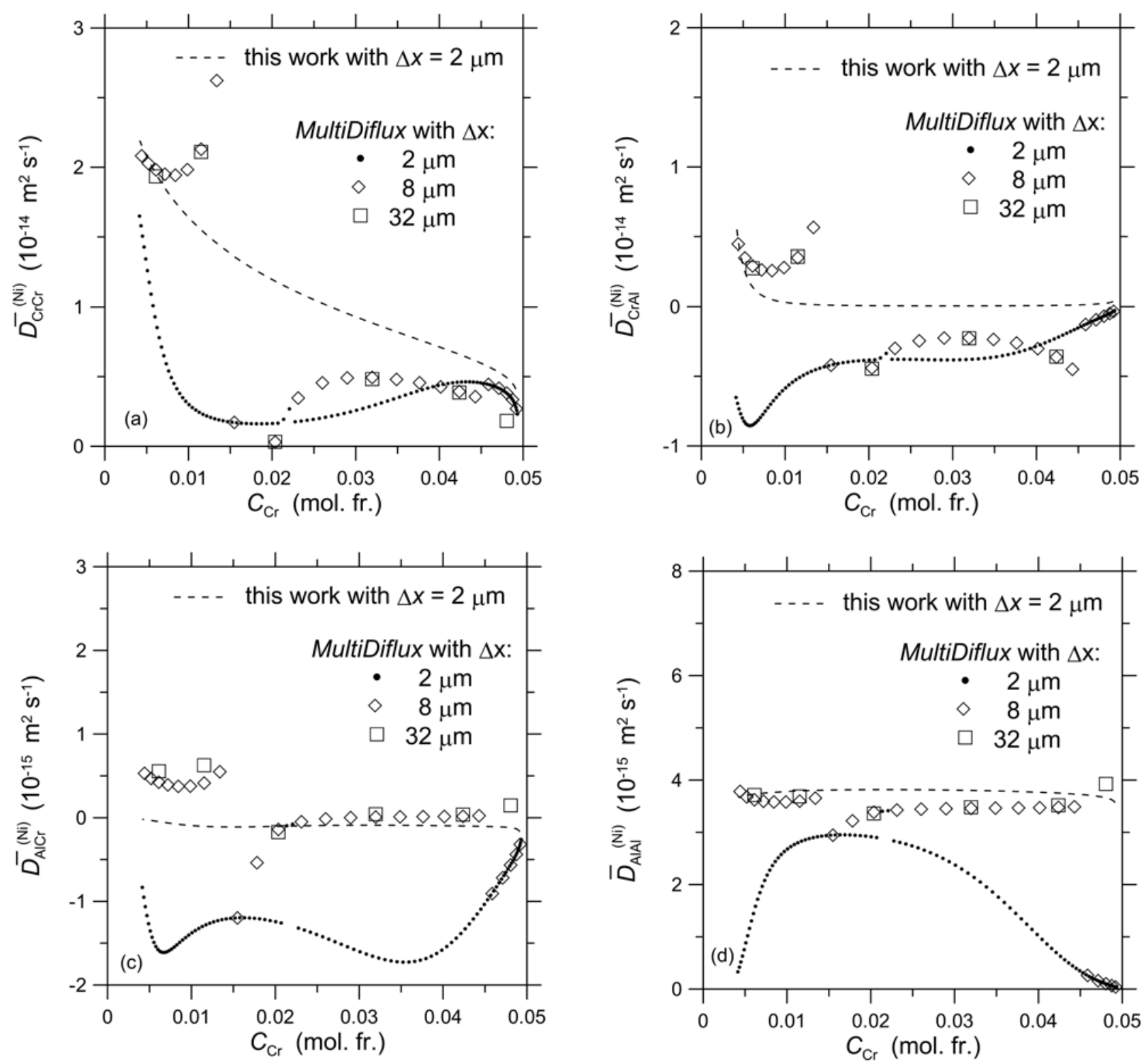

Fig. 5. Influence of $\Delta x$ on values of $\bar{D}_{i j}^{(k)}\left(C_{\mathrm{Cr}}\right)$ calculated by MultiDiFlux; fit 1. Values calculated by the proposed method (for fit 1 and $\Delta x=2 \mu \mathrm{m}$ ) are also plotted for comparison (dashed line).

shown in Fig. 6 - compare discrepancies between our results and those from MultiDiFlux in Figs. 2 and 3. This is caused by the nature of the problem: carefully measured concentration profiles lead to the considerable uncertain diffusion coefficients but, in the reversed sense, roughly estimated diffusivities produce concentration distribution with an accuracy sufficient for most practical purposes.

\section{Discussion}

The proposed method is a simple algorithm that gives estimated values of ternary interdiffusion coef- ficients, $\bar{D}_{i j}^{(3)}\left(C_{i}\right)$, from measured concentration distribution in a single diffusion couple. It needs no external measurements or additional data (Kirkendall markers shift, thermodynamic data, etc.). In terminal parts of diffusion zone, the calculated values are close to approximate coefficients obtained by HM. For quasi-binary interdiffusion (two concentrations vary, and the third one is relatively constant), the diagonal coefficients $\bar{D}_{i i}^{(3)}\left(C_{i}\right)$ approach the coefficients of mutual diffusion $D_{i}\left(C_{i}\right)$ calculated by BM.

The coefficients $\bar{D}_{i j}^{(3)}\left(C_{i}\right)$ represent the constant average values over individual intervals $\Delta x$. It was shown that selection of sufficiently low $\Delta x$ (down to about $2 \mu \mathrm{m}$ ) offers a possibility to approximate 

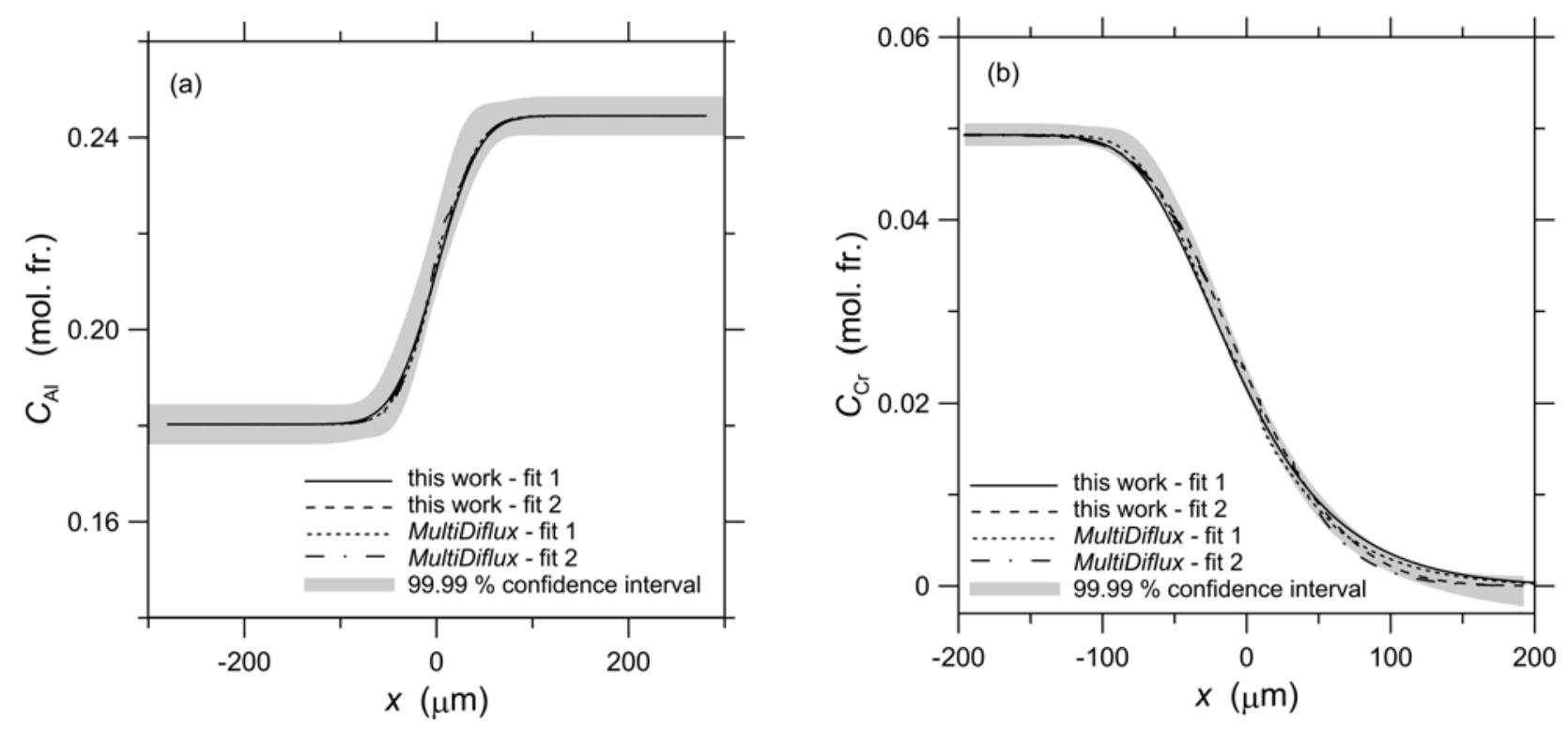

Fig. 6. Concentration profiles $C_{i}(x)$ calculated by profiler using $\bar{D}_{i j}^{(\mathrm{Ni})}\left[C_{\mathrm{Cr}}(x)\right]$ obtained by the proposed method and by the MultiDiFlux: (a) - Al, (b) - Cr.

the concentration-dependent true interdiffusion coefficients $\tilde{D}_{i j}^{(3)}\left(C_{i}\right)$ closely with the average coefficients $\bar{D}_{i j}^{(3)}\left(C_{i}\right)$. Dependence of diffusion coefficients $\bar{D}_{i j}^{(3)}\left(C_{i}\right)$ on $\Delta x$ is much weaker and more stable compared with values calculated by MultiDiFlux in a broad interval of $\Delta x=2-32 \mu \mathrm{m}$. For $\Delta x \geq 32 \mu \mathrm{m}$, our results are close to those obtained by MultiDiFlux. Differences between values of $\bar{D}_{i j}^{(3)}\left(C_{i}\right)$ calculated by both methods originate most likely in lower accuracy of MultiDiFlux calculus and/or in its inappropriate selection of a fitting function.

It should be mentioned that the present method cannot be applied to the evaluation of diffusion coefficients if $C_{1}$ is (almost) linearly dependent on $C_{2}$. In such a case, the rank of the matrix of the system of equations for $D$ 's decreases and the system cannot be solved. In other words, the diffusion couple with $C_{2} \approx r C_{1}+s(r, s$ are constants) cannot be used for reliable evaluation of $D$ 's. This limitation is close to a requirement that composition vector should be sufficiently different from eigenvector of diffusion matrix $[27]$.

\section{Summary}

A numerical method is proposed for the estimation of concentration dependent ternary interdiffusion coefficients $\bar{D}_{i j}^{(3)}\left(C_{i}\right)$ from concentration distributions $C_{1}$ and $C_{2}$ measured in a single diffusion couple.

The numerical stability and reliability of coefficients $\bar{D}_{i j}^{(3)}\left(C_{i}\right)$ were tested using the diffusion couples (Ni)-Al-Cr [14].
Our results obtained with the proposed method agree reasonably with results obtained by other methods (BM, HM) and are also close to the results from MultiDiFlux when sufficiently large intervals $\Delta x$ are used in the diffusivity calculations. Reverse reconstruction of concentration profiles with the help of interdiffusion coefficients found by the method agrees very well with the originally measured $C_{i}$ 's.

It was shown that a careful smoothing of measured concentration profiles is very important. Even a slight variation in $C_{i}(x)$ leads to a considerable change in resulting $D$ 's.

The method does not need any external characteristics such as the velocity of inert markers, thermodynamic data, etc.

\section{Acknowledgement}

This work was supported by the project CEITEC CZ.1.05/1.1.00/02.0068.

\section{References}

[1] Boltzmann, L.: Ann. Phys.-Leipzig, 53, 1894, p. 959. doi:10.1002/andp.18942891315

[2] Matano, C.: Jpn. J. Phys., 8, 1933, p. 109.

[3] Hall, L. D.: J. Chem. Phys., 21, 1953, p. 87.

[4] Morral, J. E.: Metall. Mater. Trans. A, 43, 2012, p. 3462. doi:10.1007/s11661-011-1026-z

[5] Kirkaldy, J. S., Young, D. J.: Diffusion in the Condensed State. London, Institute of Metals 1987.

[6] Darken, L. S.: Trans. AIME, 175, 1948, p. 184. 
[7] Ram-Mohan, L. R., Dayananda, M. A.: J. Phase Equilibria and Diffusion, 6, 2006, p. 566. doi:10.1361/154770306X153576

[8] Liu, D. D., Zhang, L. J., Du, Y., Xu, H. H., Jin, Z. P.: J. Alloys Compd., 566, 2013, p. 156. doi:10.1016/j.jallcom.2013.03.024

[9] Xu, H. X., Chen, W. M., Zhang, L. J., Du, Y., Tang, Y. C.: J. Alloys Compd., 644, 2015, p. 687. doi:10.1016/i.jallcom.2015.05.030

[10] Liu, D. D., Zhang, L. J., Du, Y., Jin, Y. P.: J. Alloys Compd., 634, 2015, p. 148. doi:10.1016/j.jallcom.2015.01.267

[11] Czerhati, C., Ugaste, U., van Dal, M. J. H., Lousberg, N. J. H. G. M., Kodentsov, A. A., van Loo, F. J. J.: Defect Diff. Forum, 194-199, 2001, p. 189. doi:10.4028/www.scientific.net/DDF.194-199.189

[12] Ugaste, U., Kodentsov, A. A., van Loo, F. J. J.: Phys. Met. Metallogr., 114, 2013, p. 54. doi:10.1134/S0031918X13010122

[13] Day, K. M., Ram-Mohan, L. R., Dayananda, M. A.: J. Phase Equilibria and Diffusion, 26, 2005, p. 579. doi:10.1361/154770305X74377

[14] Cermak, J., Rothova, V.: Acta Mater., 51, 2003, p. 4411. doi:10.1016/S1359-6465(03)00276-3

[15] Dayananda, M. A., Ram-Mohan, L. R.: (2004) MultiDiFlux. (Version 1.2). [Software]. https://engineering.purdue.edu/MSE/Research/ MultiDiFlux/index.html (Sept $\left.21^{\text {st }}, 2015\right)$.
[16] Dayananda, M. A.: J. Phase Equilibria and Diffusion, 26, 2005, p. 441. doi:10.1361/154770305X66493

[17] Dayananda, M. A.: Defect Diff. Forum, 297-301, 2010, p. 1451. doi:10.4028/www.scientific.net/DDF.297-301.1451

[18] Dayananda, M. A., Sohn, Y. M.: Metall. Mater. Trans. A, 30, 1999, p. 535. doi:10.1007/s11661-999-0045-5

[19] Sauer, F., Freise, Z.: Electrochem., 66, 1962, p. 353. doi:10.1002/bbpc.19620660412

[20] den Broeder, F. J. A.: Scripta Metall. Mater., 3, 1969, p. 321. doi:10.1016/0036-9748(69)90296-8

[21] Systat Software Inc: TableCurve 2D ${ }^{\circledR}$. (Version 5.01). [Software]. http://www.sigmaplot.com (Sep 21 $1^{\mathrm{st}}$, 2015).

[22] Lapin, J.: Intermetallics, 14, 2006, p. 1417. doi:10.1016/i.intermet.2006.01.048

[23] Lapin, J.: Kovove Mater., 40, 2002, p. 209.

[24] Lapin, J., Bajana, O.: Kovove Mater., 43, 2005, p. 169.

[25] Bouchet, R., Mevrel, R.: Acta Mater., 50, 2002, p. 4887. doi:10.1016/S1359-6454(02)00358-0

[26] Kirkaldy, J. S.: Isothermal Diffusion in Multicomponent Systems. In: H. Herman (Ed.), Adv. Materials Res.. New York, Interscience Publisher 1970.

[27] Jaques, A. V., LaCombe, J. C.: J. Phase Equilib. Diff., 33, 2012, p. 181. doi:10.1007/s11669-012-0028-x 